\title{
Numerical Simulation of Boundary-Layer Transition
}

\section{P.R. Spalart}

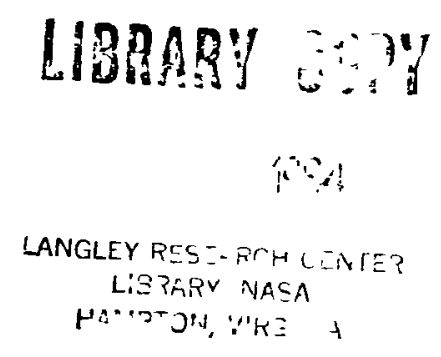


NASA Technical Memorandum 85984

\section{Numerical Simulation of Boundary-Layer Transition}

P R Spalart, Ames Research Center, Moffett Fıeld, Calıfornı 
P. R. Spalart

NASA Ames Research Center

Moffett Field, CA 94035 USA

\section{FORMULATION}

The transition to turbulence in boundary layers is investigated by direct numerical solution of the nonlinear, three-dimensional, incompressible Navier-Stokes equations in the half-infinite domain over a flat plate. Perlodicity is imposed in the $x$-direction (streamwise) and in the z-direction (spanwise); the $y$-coordinate extends from 0 to $\infty$.

The simulation is periodic in the x-direction, unlike the experiments in which the boundary-layer thickness grows in the $x$-direction. A forcing term is added to the $x$-momentum equation that approximates the convection terms associated with this spatial growth. The approximation is based on boundary-layer assumptions (applied only to the mean flow) and the self-similarity of the mean-velocity profile. With this forcing applied, the laminar velocity profile, instead of becoming an error function and thickening without bounds, is a Blastus profile. Thus, the stability characteristics are very close to the experimental characteristics. Furthermore, the equation can be written in a moving reference frame, so that the boundary-layer thickens in time while retaining a Blasius profile. The procedure of adding a forcing term allows the disturbances to extract energy from the mean flow, and is much preferable to a procedure in which the mean-velocity profile would be imposed.

The spatial representation is spectral in all directions [1]. The basis functions that are used represent divergence-free velocity fields and satisfy the boundary conditions as suggested by Leonard and Wray [2]. Leonard and Wray applied a weak formulation, which eliminates the pressure and allows an accurate and straightforward tIme-advance scheme. Leray's weak formulation is used here [3]. An advantage of this formulation over Leonard's is that it keeps the numerical Stokes operator real, symmetric, and negative-definite. On the other hand, Chebyshev polynomials cannot be used.

The $x$ - and $z$-directions are treated by Fourler serles. In the $y$-direction, the velocity field is first split into "irrotational" and "vortical" components in order to better accommodate two different length scales. The length scale of the vortical component is $\delta$, the thickness of the boundary layer. The length scale of the irrotational components is $\Lambda$, the wavelength in the $(x, z)$ plane, which is significantly larger than $\delta$. This irrotational component can be represented by a single exponential function for each horizontal wave-vector. To represent the vortical component, an exponential mapping is applied from $[0, \infty[$ into $[0,1[$, and shifted Jacobi polynomials are used in the transformed coordinate. The vortical component is infinitely differentiable over the closed interval $[0,1]$, so that the convergence of the polynomial method will be faster than algebralc. The cost of the transforms from 
real space to Jacobi space is of the order of $\mathrm{N}^{2}$. Figure 1 is a plot of the first few basis-functions versus $y$. All the functions decay exponentially as $y \rightarrow \infty$ but the first function, which includes the irrotational component, decays much more slowly than the other ones.

The time-advance scheme is hybrid and second-order accurate. The convection terms are treated by a Runge-Kutta scheme which is explicit, third-order accurate, and conditionally stable; the Stokes terms are treated by the Crank-Nicolson scheme.

\section{RESULTS}

In order to check the convergence of the method, the Orr-Sommerfeld equation was solved for a Blasius profile and for a real wave-number. This problem is known to produce a few discrete eigenvalues and a continuous spectrum on the $C_{r}=1, C_{i}<0$ axis [4]. Figure 2 is a contour plot of the error in the principal discrete eigenvalue as a function of $\mathrm{Yo}_{0}$, the length scale of mapping, and $\mathrm{Ny}$, the number of points in the $y$-direction. The convergence as $N y \rightarrow \infty$ with Yo fixed is very fast. It is expected to be faster than algebraic, but not as fast as exponential [5]. The plot also indicates the optimum value of Yo: about $2 \delta^{*}$. Figure 3 shows that the numerical spectrum includes a string of efgenvalues that becomes denser and tends to the $C_{r}=1, C_{1}<0$ axis. Its convergence is much slower than that for the discrete eigenvalues; the reason is that the corresponding eigenfunctions behave like sine waves as $\mathrm{y} \rightarrow \infty$, which makes them hard to approximate with the expansion functions in Fig. 1.

The early nonlinear stages of transition of a Blasius boundary layer, disturbed by a vibrating ribbon, were then simulated in three dimensions. A two-dimensional Tollmein-Schlichting (TS) wave of fincte amplitude was introduced in the initial field, as well as three-dimensional white nolse of much lower energy. The streamwise period was twice that of the TS wave; the spanwise period was chosen much longer to avold constralning the spectrum. Spanwise lines of particles were introduced, near the critical layer, to simulate the smoke lines used in experiments.

Figure 4 summarizes the time-evolution of the flow. The energy of the fundamental TS wave and the energy carried by all the other wave-vectors are plotted separately. The TS wave grows from branch I to branch II of the TS stability diagram, then starts decaying. The energy of the other modes remains small until after the flow crosses branch II; then it grows very rapidly, and nonlinear interactions take place. The shape factor $H$ of the boundary layer remains at the Blasius value of 2.6 until transition occurs; then 1 t rapidly decreases. The agreement with Kachanov's experiments is excellent [6].

Simulations were conducted with the same background noise, but different values for the TS wave amplitude. Figure 5 contains top views of the particles in the boundary layer. If the maxımum TS wave amplitude is less than $0.3 \%$, transition does not occur. In Fig. 5(a), with an amplitude of $0.9 \%$, three-dimensional breakdown occurs and is of the subharmonic or " $\mathrm{H}$ " type (the lambda-shaped particle lines are staggered). 
In Fig. 5(b), with amplitude 5\%, the lambda patterns are not staggered, Indicating a Klebanoff-type breakdown. The patterns appear "broken," a result of the randomness of the inftial three-dimensional disturbance. The qualitative agreement with Saric's experiments is good [7].

Figure 6 is a plot of the spectrum in an $(x, z)$ plane at the beginning of an H-type breakdown. The fundamental TS wave still dominates the spectrum; its higher harmonic is also present. The growing three-dimensional subharmonic component is obvious; the wave number and the broadband character of the Instability agree very well with Herbert's small disturbance theory [8].

\section{REFERENCES}

1. Gottlieb, D. and Orszag, S. A., "Numerical Analysis of Spectral Methods," NSF-CMBS Monograph No. 26, Society of Industrial and Applied Mathematics, Philadelphia, Penn., 1977.

2. Leonard A. and Wray, A., "A New Numerical Method for the Simulation of ThreeDimensional Flow in a Pipe," NASA TM-84267, 1982.

3. Temam, R., "Navier-Stokes Equations and Nonlinear Functional Analysis," NSF-CMBS Monograph No. 41, Society of Industrial and Applied Mathematics, Philadelphia, Penn., 1983.

4. Grosch, C. E. and Salwen, H., "The Continuous Spectrum of the Orr-Sommerfeld Equation. Pt. 1. The Spectrum and the Elgenfunctions," J. Fluid Mech., Vol. 87, Pt. 1, 1978, pp. 33-54.

5. Boyd, J. P., "The Optimization of Convergence for Chebyshev Polynomial Methods in an Unbounded Domain," J. Comp. Phys., Vol. 45, No. 1, 1982, pp. 43-79.

6. Kachanov, Y. S. and Levchenko, V.Y., "The Resonant Interaction of Disturbances at Laminar-Turbulent Transition in a Boundary Layer," J. Fluid Mech., Vol. 138, 1984, pp. 209-247.

7. Sar1c, W. S., Kozlov, V. V., and Levchenko, V. Y., "Forced and Unforced Subharmonic Resonance in Boundary-Layer Transition," AIAA Paper 84-0007, 1984.

8. Herbert, T., "Analysis of the Subharmonic Route to Transition in Boundary Layers," AIAA Paper 84-0009, 1984.

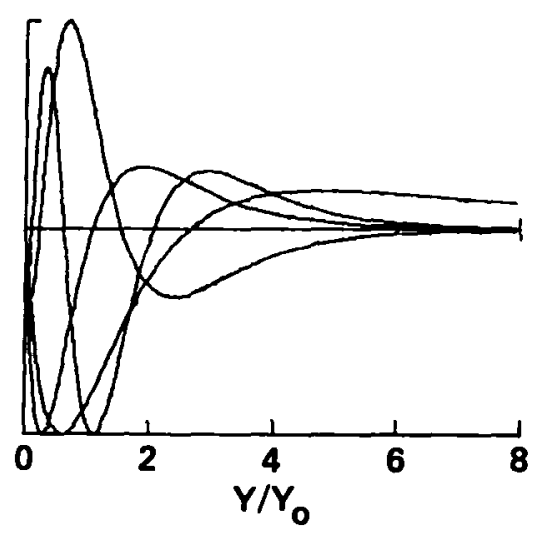

Fig. 1 First four basis-functions.

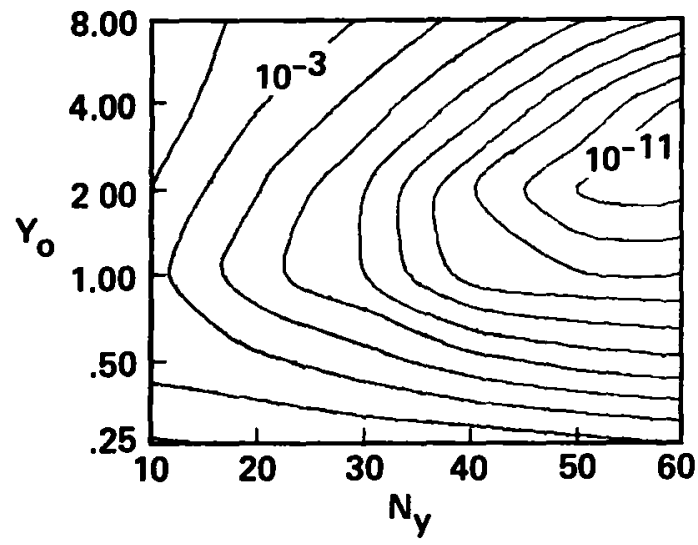

F1g. 2 Error in Orr-Sommerfeld eigenvalue. 


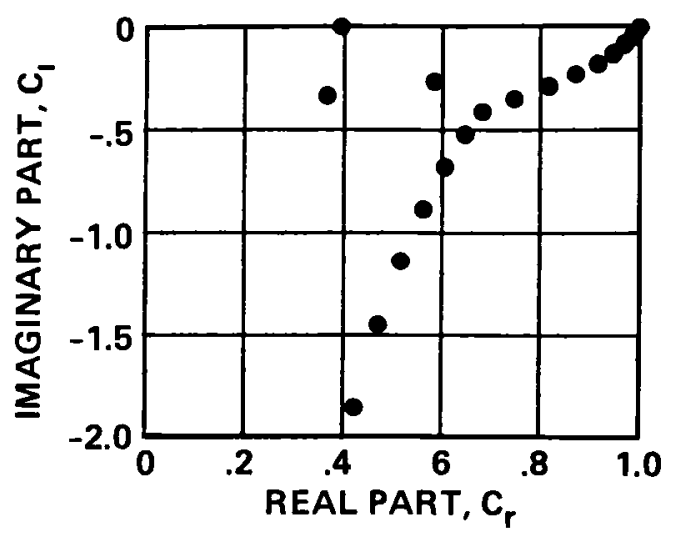

(a) $\mathrm{Ny}=50$.

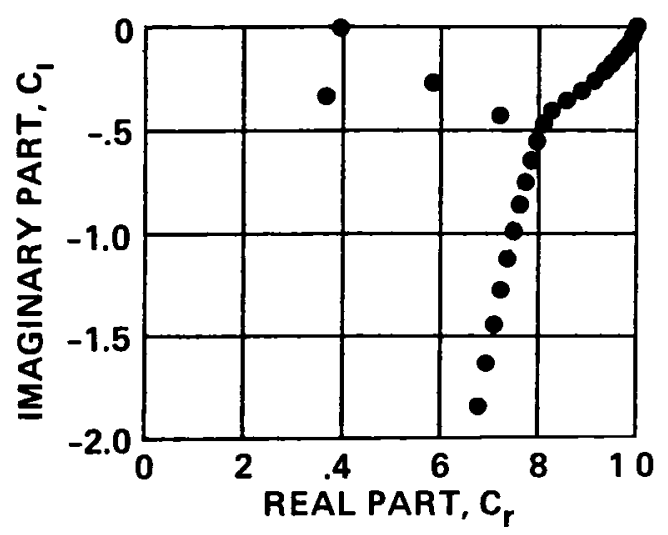

(b) $\mathrm{Ny}=100$.

Fig. 3 Numerical spectrum: Yo $=1.5$.

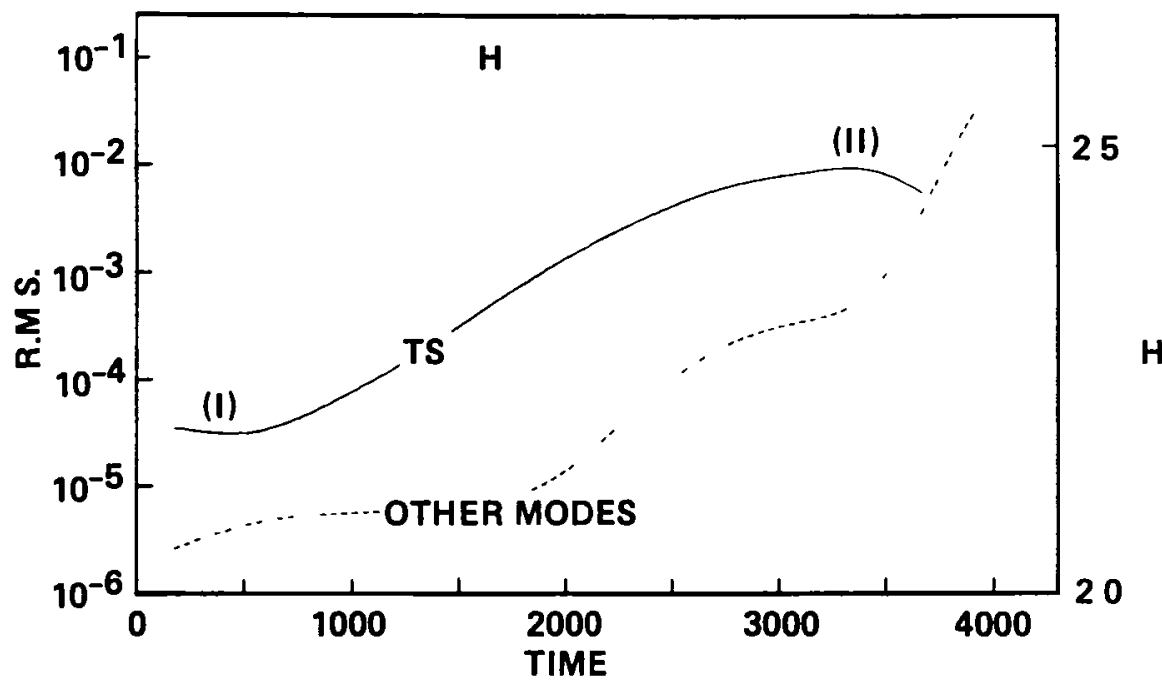

Fig. 4 Time-evolution of the disturbance energy and of the shape factor.

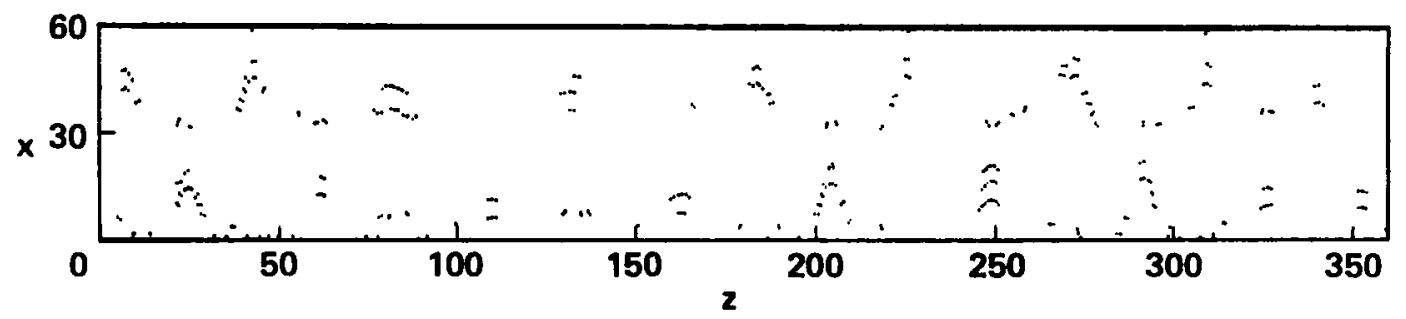

(a) Amplitude $=0.9 \%$.

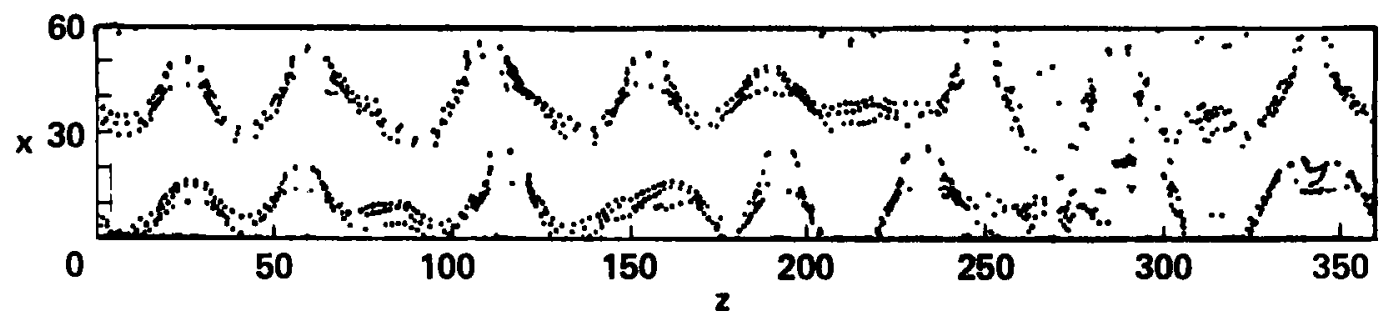

(b) Amplitude $=5 \%$.

Fig. 5 Smoke lines in transitioning boundary layer. 


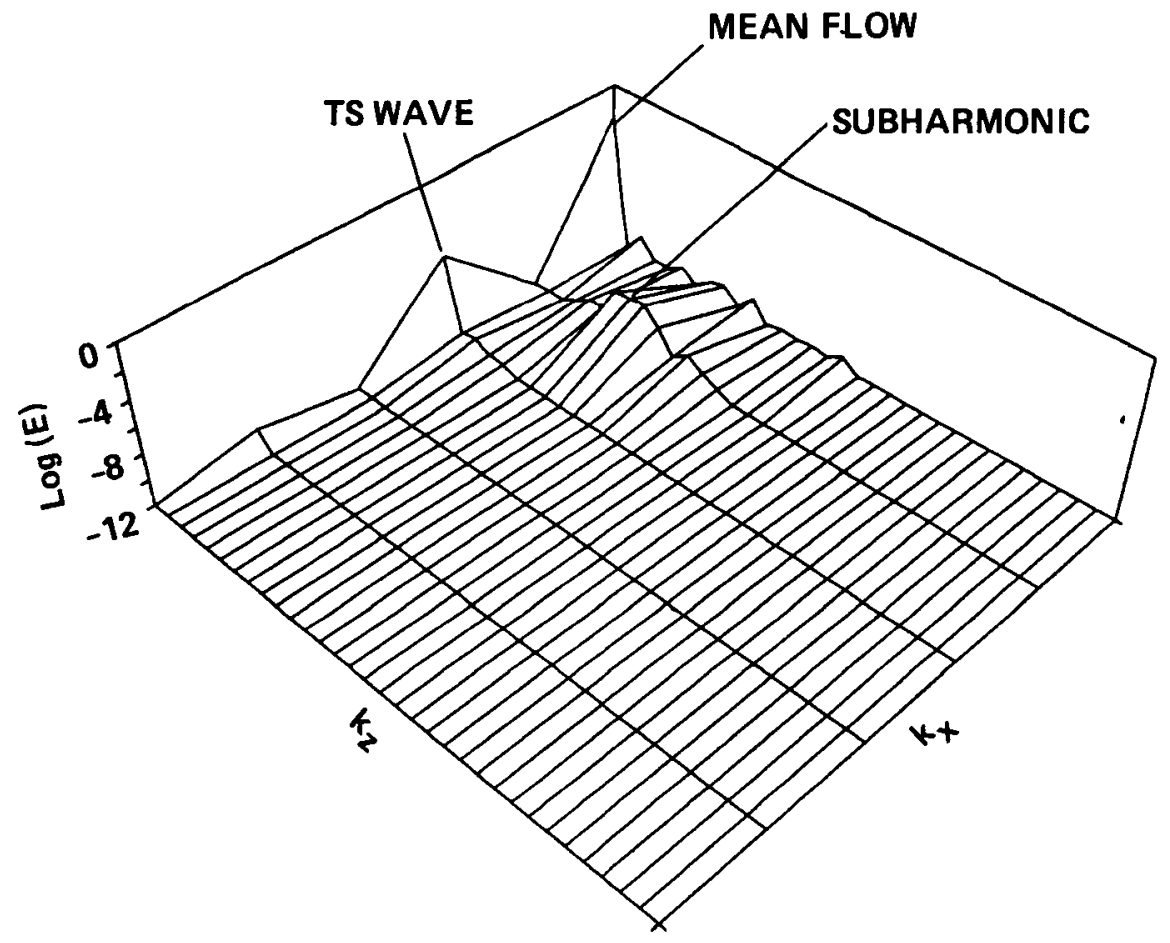

Fig. 6 Two-dimensional spectrum. 


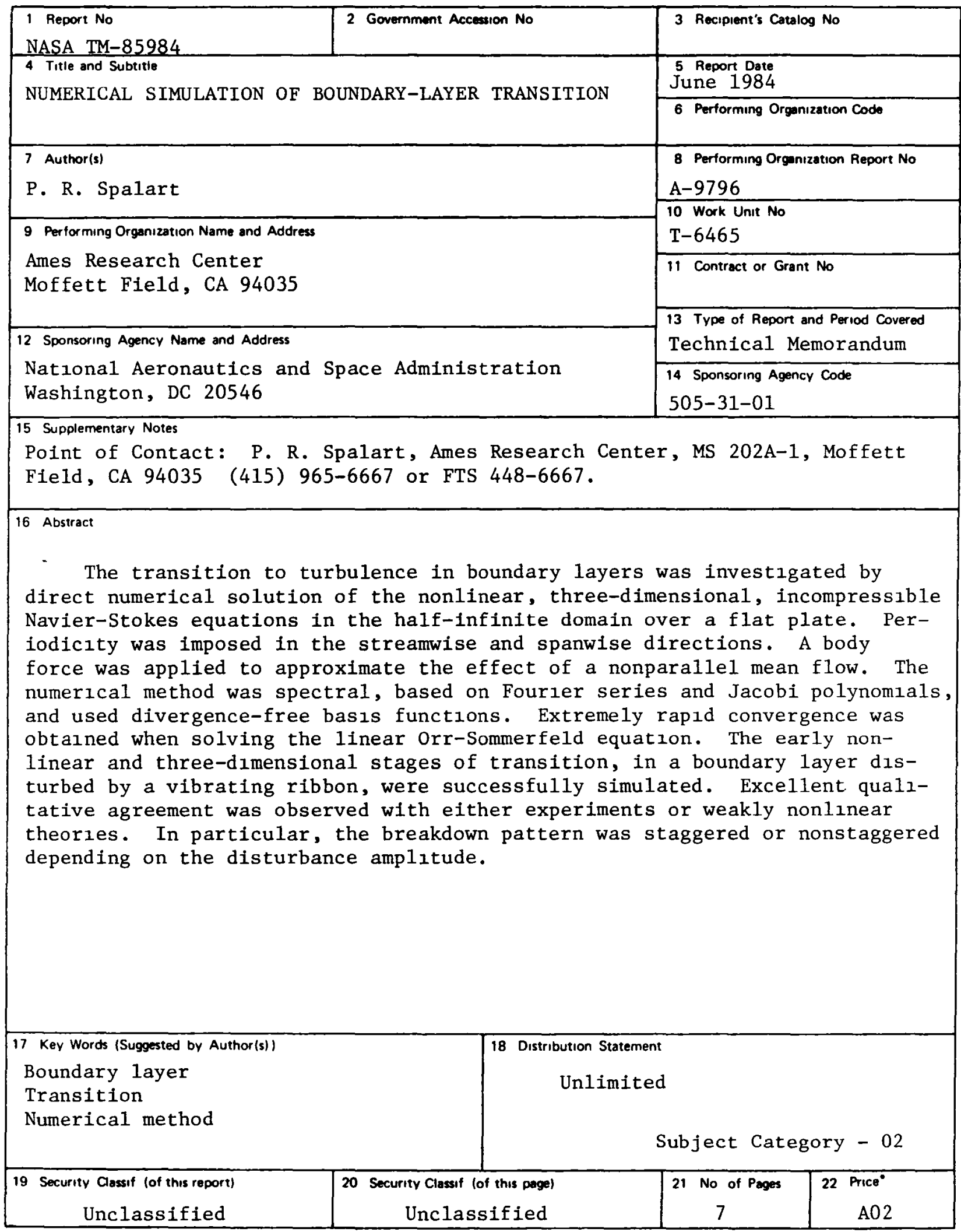

-For sale by the National Tochnical Information Service, Springfield, Virginia 22161 
End of Document 